SU-ITP-95-8

IEM-FT-86/95

SUSSEX-AST-95/4-2

gr-qc/9504022

October 9, 2018

\title{
Stationary solutions in Brans-Dicke stochastic inflationary cosmology
}

\author{
Juan García-Bellido円 \\ Astronomy Centre, School of Mathematical and Physical Sciences, \\ University of Sussex, Brighton BN1 9QH, UK. \\ Andrei Linde $]^{2]}$ \\ Department of Physics, Stanford University, \\ Stanford, CA 94305-4060, USA
}

\begin{abstract}
In Brans-Dicke theory the Universe becomes divided after inflation into many exponentially large domains with different values of the effective gravitational constant. Such a process can be described by diffusion equations for the probability of finding a certain value of the inflaton and dilaton fields in a physical volume of the Universe. For a typical chaotic inflation potential, the solutions for the probability distribution never become stationary but grow forever towards larger values of the fields. We show here that a non-minimal conformal coupling of the inflaton to the curvature scalar, as well as radiative corrections to the effective potential, may provide a dynamical cutoff and generate stationary solutions. We also analyze the possibility of large nonperturbative jumps of the fluctuating inflaton scalar field, which was recently revealed in the context of the Einstein theory. We find that in the Brans-Dicke theory the amplitude of such jumps is strongly suppressed.
\end{abstract}

\footnotetext{
${ }^{1}$ E-mail: j.bellido@sussex.ac.uk

2 E-mail: linde@physics.stanford.edu
} 


\section{Introduction}

After fifteen years of development of inflationary cosmology, the basic principles of this theory seem to be well understood, see e.g. Ref. [1]. However, there is still a very long way from these basic principles to the final theory. One of the main problems is the absence of the final version of the underlying particle theory. Fortunately, many properties of inflationary cosmology are very stable with respect to the change of its particular realization. In particular, most inflationary models predict a flat Universe $(\Omega=1)$ with a nearly scale-independent spectrum of density perturbations. Still some important deviations from the standard lore may appear when one goes from one theory of elementary particles to another. For example, in a certain class of theories, one can obtain an open [2] or a closed [3, 4] Universe, or even a Universe consisting of different causally disconnected regions with all possible values of $\Omega<1$ [4]. It would be important to find out whether some other modifications of the inflationary paradigm may appear when one implements it in different theories of fundamental interactions.

An interesting playground for testing the robustness of various ideas about inflation is provided by the Brans-Dicke theory [5]. It remains to be seen whether this theory or some of its generalizations can have a sufficiently good motivation, e.g. from the point of view of string theory [6]-[8]. In any case, however, some qualitatively new effects which appear in the BD inflationary cosmology may justify its investigation.

One of these effects is the possibility to avoid the graceful exit problem of the first-order inflation [9]. This possibility, however, requires the introduction of an effective potential and/or a nonminimal kinetic term for the Brans-Dicke field [10], which makes the corresponding theory

rather complicated and may lead to certain problems [11]. In some of the recent versions of these models the end of inflation occurs in the standard way, during the stage of slow rolling [12].

In what follows we will be interested in another specific effect which may appear in the inflationary Brans-Dicke cosmology. In this theory quantum fluctuations of the Brans-Dicke field $\phi$ during inflation driven by the inflaton field $\sigma$ lead to the division of the Universe into different exponentially large regions where the effective gravitational constant $G=M_{\mathrm{P}}^{-2}=\frac{\omega}{2 \pi \phi^{2}}$ can take all possible values from 0 to $\infty$, see Ref. [13].

This effect becomes especially interesting if one takes into account the process of self-reproduction of inflationary universe. This process can be studied in a most adequate way by using the stochastic approach to inflation and investigating the probability distribution $P_{p}(\phi, \sigma, t)$, which gives the relative fraction of the volume of the Universe containing the inflaton field $\sigma$ and the 
Brans-Dicke field $\phi$ at the moment $t$. A detailed study of the distribution $P_{p}$ was performed recently in Ref. [14], where it was shown that in many inflationary models based on the Einstein theory of gravity (i.e. the theory with a constant Brans-Dicke field) this probability distribution rapidly approaches a stationary regime. This means that if one takes a section of the Universe at a given time $t$ and calculates the relative fraction of domains of the Universe with given properties, the result will not depend on the time $t$, both during inflation and after it.

However, the stationary character of the probability distribution $P_{p}$ in general relativity is closely related to the existence of the Planck boundary, where the potential energy density $V(\sigma)$ becomes comparable with the Planck density $M_{\mathrm{P}}^{4}$. Typically the distribution $P_{p}(\sigma, t)$ rapidly moves towards large $\sigma$, for the reason that the volume of domains with large $V(\sigma)$ grows very fast. The distribution $P_{p}(\sigma, t)$ becomes stabilized as it approaches the Planck boundary, where, as it is argued in [14]-16, the process of self-reproduction of inflationary domains is less efficient, or inflation becomes impossible altogether.

Meanwhile, in the Brans-Dicke theory the situation is more complicated [17, 18]. Since the effective Planck mass in this theory depends on $\phi, M_{\mathrm{P}}^{2}(\phi)=\frac{2 \pi}{\omega} \phi^{2}$, the Planck boundary instead of being a point becomes a line $\phi^{4}=\frac{\omega^{2}}{4 \pi^{2}} V(\sigma)$. Then the distribution $P_{p}(\phi, \sigma, t)$ first moves towards the Planck boundary, and after that it slides along this boundary in the direction where the rate of expansion of the Universe becomes greater. Such runaway solutions typically lead to the distributions $P_{p}$ rapidly moving towards indefinitely large values of $\phi$ and $\sigma$. As a consequence, a typical value of the Planck mass at the end of inflation also becomes indefinitely large, and the effective gravitational constant vanishes.

In fact, runaway solutions may appear even in the ordinary Einstein theory. This may happen if the inflaton field is nonminimally coupled to curvature due to the existence of the interaction $-\frac{1}{2} \xi \sigma^{2} R$, with $\xi<0$ [16. On the other hand, in the theories with $\xi>0$ one may encounter many different regimes. Depending on the value of $\xi$, one may not have inflation at all, or one may have inflation without self-reproduction of inflationary domains, or inflation and self-reproduction with a stationary distribution $P_{p}$.

This suggests us to study a more general version of the Brans-Dicke theory, where not only the Brans-dicke field $\phi$, but also the inflaton field $\sigma$ is nonminimally coupled to gravity. Some models of this type have been discussed before in [19, 20]. Note that this framework seems to be much more natural than the standard one, where it is assumed that only one of the two scalar fields (the Brans-Dicke field) is nonminimally coupled to gravity.

This paper is organized as follows. In Section 2 we discuss the problem of runaway solutions 
in the Brans-Dicke stochastic inflation. In Section 3 we derive a set of equations for inflation in the Brans-Dicke theory with a non minimally coupled inflaton scalar field. We study the case of a purely massive scalar field and find that it provides a natural cutoff for the motion of the inflaton and dilaton fields, resulting in the existence of stationary solutions to the diffusion of both fields. In order to test the stability of the results, we analyse the same model in the presence of a non zero self-coupling of the inflaton. We find that such a term is enough to destroy the stationarity of the probability distribution, making such a stationarity rather improbable. In Section 3.1 we propose a different solution to the runaway behavior of the scalar fields by considering 1-loop corrections to the effective potential. For certain values of the parameters of the model, it is possible that the effective potential of the inflaton field acquires a maximum at exponentially large values of $\sigma$. This provides a natural cutoff for the rate of inflation and makes the distribution $P_{p}$ stationary. Furthermore, the Planck mass in this model is exponentially larger than the only scale in the problem, the mass of the inflaton, thus naturally explaining a hierarchy of scales. In Section 5 we explore the possibility of very large quantum jumps of the scalar field [21] in the context of the Brans-Dicke theory. In Section 6 we draw some conclusions.

\section{Runaway solutions in Brans-Dicke inflation}

In this section we will introduce the problem of runaway solutions in Brans-Dicke cosmology. For a detailed analysis see Refs. [17, 18]. Consider the evolution of the inflaton field $\sigma$ with a generic chaotic potential in a JBD theory of gravity with dilaton field $\phi$,

$$
\mathcal{S}=\int d^{4} x \sqrt{-g}\left[\frac{1}{8 \omega} \phi^{2} R-\frac{1}{2}(\partial \phi)^{2}-\frac{1}{2}(\partial \sigma)^{2}-V(\sigma)\right],
$$

where Planck mass is written in terms of the dilaton field as $M_{\mathrm{P}}^{2}(\phi)=\frac{2 \pi}{\omega} \phi^{2}$. For generic inflaton potentials of the type $V(\sigma)=\frac{\lambda}{2 n} \sigma^{2 n}$, the equations of motion for the homogeneous fields in the slow-roll approximation are

$$
\frac{\dot{\phi}}{\phi}=\frac{H}{\omega}, \quad \frac{\dot{\sigma}}{\sigma}=-\frac{n}{2} \frac{H}{\omega} \frac{\phi^{2}}{\sigma^{2}}
$$

This implies that

$$
\frac{d}{d t}\left(\phi^{2}+\frac{2}{n} \sigma^{2}\right)=0
$$

which means that the fields move along a circle of radius $r^{2}=\phi^{2}+\varphi^{2}$, with $\varphi^{2}=\frac{2}{n} \sigma^{2}$, see Ref. [13]. Inflation begins at the Planck boundary $\phi^{2}=\frac{\omega \sqrt{\lambda}}{2 \pi \sqrt{2} n} \sigma^{n}$ and ends somewhere at the line $\phi^{2} \simeq \frac{\omega}{2} \sigma^{2} \gg \sigma^{2}$. This implies that if inflation begins at $\sigma_{0} \gg \phi_{0}$, it will end at $\phi \sim \sqrt{\frac{2}{n}} \sigma_{0}$. Thus, if we find a reason why $\sigma_{0}$ should be large, we will be able to explain why $M_{\mathrm{P}}=\sqrt{\frac{2 \pi}{\omega}} \phi \sim 2 \sqrt{\frac{\pi}{n \omega}} \sigma_{0}$ is also large. 
This simple picture becomes much more complicated if one takes into account that in addition to the classical motion, the fields $\phi$ and $\sigma$ experience quantum jumps with a typical amplitude $\frac{H}{2 \pi}$ during each time interval $H^{-1}$. One can easily verify that (for $\phi \ll \sigma$ ) these jumps are greater than the classical rolling of the fields for

$$
\frac{3 \pi^{2}}{\omega^{3}} \phi^{4}<V(\sigma)<\frac{4 \pi^{2}}{\omega^{2}} \phi^{4},
$$

the last inequality corresponding to the Planck boundary. Thus, if the field is not very far away from the Planck boundary (but still sufficiently far away so that our approach remains reliable), the motion of the field occurs not due to classical rolling but due to Brownian jumps in all possible directions. The jumps in the direction of greater Hubble constant lead to much more rapid expansion of space. As a result, very soon (in the synchronous time $t$ ) the main part of the physical volume of the Universe shifts towards the Planck boundary where the Hubble constant takes its greatest values. After that the field $\sigma$ can take all possible values along the Planck boundary, and therefore the value of the field $\phi$ in the domains reaching the boundary of the end of inflation does not depend on the initial value $\sigma_{0}$.

However, the Hubble constant $H(\phi, \sigma)$ is different in different parts of the Planck boundary. If it has a maximum at some point $\sigma=\sigma_{\max }$ along the Planck boundary, then the main part of the volume of the Universe will be produced as a result of exponentially rapid inflation near this point. Therefore the main part of the volume of the Universe after inflation will be produced with $M_{\mathrm{P}} \sim \sqrt{\frac{4 \pi}{n \omega}} \sigma_{\max }$ [17.

Unfortunately, as we have shown in [17, 18], this regime is not realized in the model (1) with $V(\sigma)=\frac{\lambda}{2 n} \sigma^{2 n}$, nor in the models with exponentially growing potentials. The reason is very simple: The Hubble parameter in such theories grows along the Planck boundary. Therefore the leading contribution to the volume of the Universe is given by the domains expanding with ever growing speed and containing indefinitely large values of the fields $\sigma$ and $\phi$. The diffusion equations describing this process have runaway solutions which describe distributions $P_{p}(\phi, \sigma, t)$ running towards infinitely large $\sigma$. In many cases, the center of the distribution $P_{p}$ reaches infinitely large values of $\sigma$ within finite time.

This does not necessarily mean that the corresponding theories are physically unacceptable. It may happen that our idea that we should live in a part of the Universe corresponding to a maximum of $P_{p}(\phi, \sigma, t)$ at a given time $t$ is incorrect; see Ref. [17] for a discussion of this issue. In particular, as it was shown in [14, 17], the behavior of the distribution $P_{p}(\phi, \sigma, t)$ depends on the choice of time parametrization. If one studies, e.g. the probability distribution $P_{p}(\phi, \sigma, \tau)$, where $\tau=\ln a(t)$, its behavior may be quite different, and it may not exhibit any runaway solutions. Moreover, it is very easy to stabilize the distribution $P_{p}(\phi, \sigma, t)$ by making the effective potential 
$V(\sigma)$ very curved and unsuitable for inflation at large $\sigma$. Still it would be interesting to study this phenomenon in a more detailed way and to see whether the distribution $P_{p}(\phi, \sigma, t)$ in the Brans-Dicke theory can be stabilized in a natural way. If this stabilization can occur only at very large values of the field $\sigma$, then the typical value of the Brans-Dicke field $\phi$ at the end of inflation will also be extremely large. This may give us a tentative explanation of the anomalously large value of the Planck mass.

\section{A more general theory}

Let us now turn to a scenario in which some of the problem discussed above could be resolved. For that purpose we will consider the classical evolution of the inflaton field with a generic chaotic potential, in the context of the Jordan-Brans-Dicke theory of gravity and a curvature coupled inflaton with a non minimal coupling $\xi$,

$$
\mathcal{S}=\int d^{4} x \sqrt{-g}\left[\frac{1}{8 \omega} \phi^{2} R-\frac{1}{2} \xi \sigma^{2} R-\frac{1}{2}(\partial \phi)^{2}-\frac{1}{2}(\partial \sigma)^{2}-V(\sigma)\right]
$$

In this theory the Planck mass takes the form

$$
M_{\mathrm{P}}^{2}(\phi, \sigma)=16 \pi \Phi=8 \pi\left(\frac{1}{4 \omega} \phi^{2}-\xi \sigma^{2}\right)
$$

where $\Phi$ plays the role of the Brans-Dicke scalar. The value of the Brans-Dicke parameter $\omega$ is bounded by the post-Newtonian experiments [22] and primordial nucleosynthesis [23] to be very large, $\omega>500$, and therefore it is reasonable to use the approximation $\omega \gg 1$ in the following analysis. The parameter $\xi$, on the other hand, is unconstrained and could take positive or negative values. Here we will consider the case of a small and negative $\xi$.

The equations of motion for the theory (5) can be written as

$$
\begin{gathered}
\nabla^{2} \phi=\frac{1}{4 \omega} \phi R, \\
\nabla^{2} \sigma=-V^{\prime}(\sigma)-\xi \sigma R, \\
-\left(\frac{1}{4 \omega} \phi^{2}-\xi \sigma^{2}\right)\left(R_{\mu \nu}-\frac{1}{2} g_{\mu \nu} R\right)=g_{\mu \nu} V(\sigma)+\left(\nabla_{\mu} \nabla_{\nu}-g_{\mu \nu} \nabla^{2}\right)\left(\frac{1}{4 \omega} \phi^{2}-\xi \sigma^{2}\right) \\
+\left(\partial_{\mu} \phi \partial_{\nu} \phi-\frac{1}{2} g_{\mu \nu}(\partial \phi)^{2}\right)+\left(\partial_{\mu} \sigma \partial_{\nu} \sigma-\frac{1}{2} g_{\mu \nu}(\partial \sigma)^{2}\right) .
\end{gathered}
$$

We can then write the exact equations for the homogeneous fields in flat space $(k=0)$ as

$$
\frac{1}{2}\left[\left(1+\frac{3}{2 \omega}\right) \nabla^{2} \phi^{2}+(1-6 \xi) \nabla^{2} \sigma^{2}\right]=4 V(\sigma)-\sigma V^{\prime}(\sigma)
$$




$$
\begin{aligned}
{\left[\frac{1}{4 \omega}\left(1+\frac{3}{2 \omega}\right) \phi^{2}-\xi(1-6 \xi) \sigma^{2}\right] R } & =4 V(\sigma)-6 \xi \sigma V^{\prime}(\sigma)-(1-6 \xi) \dot{\sigma}^{2}-\left(1+\frac{3}{2 \omega}\right) \dot{\phi}^{2} \\
\left(\frac{1}{4 \omega} \phi^{2}-\xi \sigma^{2}\right) 3 H^{2} & =V(\sigma)+6 \xi H \dot{\sigma} \sigma+\frac{1}{2} \dot{\sigma}^{2}-\frac{3}{2 \omega} H \dot{\phi} \phi+\frac{1}{2} \dot{\phi}^{2}
\end{aligned}
$$

where $R=12 H^{2}+6 \dot{H}$. Note that for $\xi=0$ and $\Phi=\phi^{2} / 8 \omega$ we recover the usual BD equations. From now on we will drop the last two terms of Eqs. (10) since they are subleading for large $\omega$.

During inflation, we can write the equations of motion of the homogeneous fields $\phi$ and $\sigma$ for small $|\xi|$ and large $\omega$, in the slow-roll approximation $\left(\ddot{\phi} \ll H \dot{\phi} \ll H^{2} \phi\right)$, as

$$
\begin{aligned}
\dot{\phi} & =\frac{H \phi}{\omega}, \\
\dot{\sigma} & =\frac{H \sigma}{\omega}\left[\left(1-\frac{\sigma V^{\prime}}{4 V}\right)\left(\frac{\phi^{2}}{\sigma^{2}}+\mu\right)-\frac{\phi^{2}}{\sigma^{2}}\right], \\
H^{2} & =\frac{\omega}{3} \frac{4 V(\sigma)}{\phi^{2}+\mu \sigma^{2}},
\end{aligned}
$$

where $\mu \equiv 4 \omega|\xi|$. Note that for small $\mu$ they reduce to the usual BD equations.

\subsection{Massive curvature-coupled inflaton}

Let us consider in some detail the theory (5) with $V(\sigma)=\frac{1}{2} m^{2} \sigma^{2}$ at large $\sigma$ and very small $\xi<0$. In this case it is useful to make a change of variables to polar coordinates $r^{2}=\phi^{2}+\varphi^{2}, z=$ $\frac{\phi}{\varphi}, \varphi^{2}=2(1+6|\xi|) \sigma^{2} \simeq 2 \sigma^{2}$. In these coordinates, the equations of motion (11) take the form

$$
\begin{aligned}
& \dot{z}=\frac{z H}{\omega}\left(z^{2}+1-\mu / 2\right), \\
& \dot{r}=\frac{r H}{\omega} \frac{\mu / 2}{1+z^{2}}, \\
& H^{2}=\frac{\omega}{3} \frac{m^{2}}{z^{2}+\mu / 2} .
\end{aligned}
$$

One can solve these equations parametrically in the $z$-variable, for $\mu \neq 2$,

$$
\begin{aligned}
& \frac{d r}{d z}=\frac{r \mu / 2}{z\left(1+z^{2}\right)\left(z^{2}+1-\mu / 2\right)}, \quad r_{o}=r\left(z_{o}\right) \\
& r(z)=r_{o}\left(\frac{z}{z_{o}}\right)^{\mu /(2-\mu)}\left(\frac{1+z^{2}}{1+z_{o}^{2}}\right)^{1 / 2}\left(\frac{z^{2}+1-\mu / 2}{z_{o}^{2}+1-\mu / 2}\right)^{-1 /(2-\mu)}
\end{aligned}
$$

which gives

$$
\begin{aligned}
& \phi(z)=r(z)\left(1+z^{-2}\right)^{-1 / 2} \\
& \varphi(z)=r(z)\left(1+z^{2}\right)^{-1 / 2} .
\end{aligned}
$$


For $|\xi| \ll 1$, the condition for existence of inflation $\left(|\dot{H}|<H^{2}\right)$ is $\dot{\sigma}^{2}<V$,

$$
\left(z^{2}-\mu / 2\right)^{2}<\frac{3 \omega}{2}\left(z^{2}+\mu / 2\right)
$$

which gives a slightly modified condition for the end of inflation, $z_{e}^{2}+\mu / 2=3 \omega / 2$.

For very small $\mu$, the trajectory is very close to a circle in the plane $(\varphi, \phi)$. However, for arbitrary $\mu$ the scalar fields move along a more complicated trajectory. There are regions of parameter space for which inflation never ends. For instance, if $\mu>2$ and $z^{2}<(\mu-2) / 2$, then $\dot{z}<0$ and inflation never ends (unless the field $\sigma$ tunnels (diffuses) to its large values quantum mechanically). This is a rather surprising result since there is nothing singular in our equations (12) at $\mu=2$. The reason of this unexpected behavior is that the field $\sigma$ in the regime $\phi \ll \sigma$ for $\mu>2$ grows much faster than the field $\phi$, so it never approaches the end of inflation with $\phi \gg \sigma$.

The value of the effective Planck mass at the end of inflation is given by (see Eq.13),

$$
M_{\mathrm{P}}=\sqrt{\frac{2 \pi}{\omega}} r\left(z_{e}\right)\left(\frac{z_{e}^{2}+\mu / 2}{z_{e}^{2}+1}\right)^{1 / 2} \simeq \sqrt{\frac{2 \pi}{\omega}} r_{o} z_{o}\left(\frac{2-\mu}{2 z_{o}^{2}}\right)^{\frac{1}{2-\mu}}
$$

where we have used the fact that $z_{e} \gg 1$ and $z_{o} \ll 1$. Note that the value of the Planck mass at the end of inflation is very sensitive to initial conditions and, for the reason explained above, it becomes exponentially large when $\mu \rightarrow 2: \quad M_{\mathrm{P}}=\sqrt{4 \pi|\xi|} \phi_{o} \exp \left(z_{o}^{-2}\right)$.

We should now discuss possible initial conditions and the regime of self-reproduction of the Universe in this model. The Planck boundary in this theory looks like an ellipse

$$
\begin{gathered}
\phi^{2}+\mu\left(\sigma-\frac{\sigma_{\max }}{2}\right)^{2}=\frac{\mu \sigma_{\max }^{2}}{4}, \\
\sigma_{\max } \equiv \frac{m}{8 \pi \sqrt{2}|\xi|} .
\end{gathered}
$$

There is no constraint on $\mu$; however, we will assume that $\xi$ and $1 / \omega$ are both of the same order of magnitude. We will see that this is a consistent approximation in this scenario.

The amplitude of quantum fluctuations of the scalar fields, $\delta \phi$ and $\delta \sigma$, whose wavelengths are stretched beyond the horizon and act on the quasi-homogeneous background fields like a stochastic force, can be computed as in 17, 18 and they are given by (for $\omega \gg 1$ and $|\xi| \ll 1$ )

$$
\delta \phi \simeq \frac{H}{2 \pi}, \quad \delta \sigma \simeq \frac{H}{2 \pi} .
$$

Density perturbations in our model can be calculated as in Refs. [17, 18, 24]. For the theory 
$V(\sigma)=\frac{1}{2} m^{2} \sigma^{2}$, in the large $\omega$ limit

$$
\frac{\delta \rho}{\rho} \sim \frac{50 m}{M_{\mathrm{P}}} .
$$

Note that the larger is the Planck mass at the end of inflation in a given region of the Universe, the smaller will be density perturbations in this region. This suggests that the large value of Planck mass $M_{\mathrm{P}}$ in our part of the Universe may be related to the small value of the amplitude of density perturbations $\frac{\delta \rho}{\rho} \sim 5 \cdot 10^{-5}$ [17]. In other words, instead of two independent small parameters, $\frac{m}{M_{\mathrm{P}}} \ll 1$ and $\frac{\delta \rho}{\rho} \sim 5 \cdot 10^{-5}$, we have only one.

As we already discussed in the previous section, those inflationary domains where quantum jumps during the time $H^{-1}$ are more important than their classical motion, enter the regime of self reproduction. One can easily show that in our case the region where this regime is possible is the interior of the ellipse

$$
\begin{gathered}
\phi^{2}+\nu\left(\sigma-\frac{\sigma_{*}}{2}\right)^{2}=\frac{\nu \sigma_{*}^{2}}{4} \\
\sigma_{*} \equiv \frac{m}{4 \pi \sqrt{6}|\xi|^{3 / 2}}, \quad \nu \equiv \mu-1+\left(\mu^{2}-\mu+1\right)^{1 / 2} .
\end{gathered}
$$

In our model the Hubble constant takes its maximal values along the line $\phi=0$ (i.e. $z=0$ ). Along this line there is a wide region where self-reproduction is possible. This region begins at the Planck boundary (17) and ends at the self-reproduction boundary (20):

$$
\frac{m}{8 \pi \sqrt{2}|\xi|}<\sigma<\frac{m}{4 \pi \sqrt{6}|\xi|^{3 / 2}} .
$$

Since this interval is limited, runaway solutions here are impossible. In such a situation the probability distribution $P_{p}(\phi, \sigma, t)$ should be stationary, with a maximum somewhere at this line, in the interval (21).

Note, however, that this maximum should be relatively smooth, since the value of the Hubble constant does not depend on $\sigma$ along the line $\phi=0$ :

$$
H(\phi=0)=\frac{m}{\sqrt{6}|\xi|^{1 / 2}} .
$$

For a complete investigation of the probability distribution $P_{p}(\phi, \sigma, t)$ and of the value of $M_{\mathrm{P}}$ at the end of inflation one should solve numerically the diffusion equations for $P_{p}(\phi, \sigma, t)$, as we did in Ref. [17. To get a rough idea of the resulting distribution of possible values of $M_{\mathrm{P}}$ at the end

\footnotetext{
${ }^{3}$ Note that during the last stages of inflation, there is an approximate equivalence of the Einstein and Jordan frames.
} 
of inflation one can simply take all points at the boundary of self-reproduction and treat them as initial conditions for the solutions (13), (16). However, one can get a much better picture if one takes into account that the main contribution to the probability distribution on this boundary is given by the part of the boundary of the region of self-reproduction with the highest value of $H$. This value is achieved for $\sigma=\sigma_{*}$ (20) at the line $\phi=0$. The peak cannot be wider than $\frac{\sqrt{\nu}}{2} \sigma_{*}$ in the $\phi$-direction, and in fact it is expected to be much more narrow. Estimates of the values of the effective Planck mass generated after inflation suggest that $M_{\mathrm{P}}$ can take all possible values from 0 to $\infty$, but typical values are much greater than $\frac{m}{\sqrt{\mu \xi}}$. If, for example, one considers the evolution of domains with initial values of the fields $\sigma \sim \sigma_{*}$ and $\phi \gtrsim \frac{H}{2 \pi}$ (due to unavoidable quantum jumps near $\phi=0$ ), Eq. (16) yields

$$
\frac{m}{M_{\mathrm{P}}} \gtrsim \sqrt{3 \pi \mu}\left(\frac{4|\xi|^{2}}{2-\mu}\right)^{\frac{1}{2-\mu}}
$$

One can then estimate the allowed values of $|\xi|$ from the amplitude of density perturbations (19). For $\mu \simeq 1$ we find $|\xi| \lesssim 4 \cdot 10^{-4}$, which is consistent with the constraint $\omega>500$ and our condition $\mu \simeq 1$. Note also that $\frac{m}{M_{\mathrm{P}}} \simeq \sqrt{6 \pi} \exp \left(-\frac{1}{2|\xi|^{2}}\right)$ for $\mu \rightarrow 2$, and it becomes very easy to obtain a very small ratio of $\frac{m}{M_{\mathrm{P}}}$ even for not too small values of $|\xi|$. Therefore, we have a natural realization of our model without the need for very small numbers. In fact one could argue that $\omega \sim 500$ is not such a large number, if we understand it as the dimensionless coupling $\beta$ of matter to the dilaton in the Einstein frame [25], $2 \beta=(2 \omega+3)^{-1 / 2} \sim 0.03$.

\subsection{Self-coupled inflaton}

We must now consider possible corrections to the inflaton potential, in order to see whether our results are stable with respect to small modifications of the theory. Let us add an inflaton self-coupling to the theory, $V(\sigma)=\frac{1}{2} m^{2} \sigma^{2}+\frac{\lambda}{4} \sigma^{4}$. The corresponding equations of motion read

$$
\begin{aligned}
& \dot{z}=\frac{z H}{\omega} \frac{2 m^{2}\left(1+z^{2}\right)\left(2 z^{2}+2-\mu\right)+\lambda r^{2}\left(1+2 z^{2}\right)}{4 m^{2}\left(1+z^{2}\right)+\lambda r^{2}}, \\
& \dot{r}=\frac{r H}{\omega} \frac{2 m^{2}\left(1+z^{2}\right) \mu-\lambda r^{2} z^{2}}{\left(4 m^{2}\left(1+z^{2}\right)+\lambda r^{2}\right)\left(1+z^{2}\right)}, \\
& H^{2}=\frac{\omega}{6} \frac{4 m^{2}\left(1+z^{2}\right)+\lambda r^{2}}{\left(1+z^{2}\right)\left(2 z^{2}+\mu\right)} .
\end{aligned}
$$

There is a bifurcation line in the plane $(z, r)$ that separates domains for which inflation never ends. This is the line $\dot{z}=0$,

$$
z^{2}=\frac{\mu-2}{2}-\frac{\lambda r^{2}}{4 m^{2}} \frac{1+2 z^{2}}{1+z^{2}}
$$


For $\mu>2$ and $r^{2}<2 m^{2}(\mu-2) / \lambda$, there are values of $z$ for which $\dot{z}<0$, which could suggest,

just as in the previous model with $V(\sigma)=\frac{1}{2} m^{2} \sigma^{2}$, that the end of inflation cannot be reached. However, as $r$ increases (24), $\dot{z}$ eventually changes sign and inflation ends.

The boundary of self-reproduction in this theory is given by

$$
\left(\phi^{2}+\nu \sigma^{2}\right)\left(1-\frac{\phi^{2}}{\sigma_{c}^{2}}\right)=\nu \sigma_{*} \sigma\left(1+\frac{\sigma^{2}}{\sigma_{c}^{2}}\right)^{1 / 2}
$$

where $\nu$ and $\sigma_{*}$ were defined in (20).

The Planck boundary for this theory is

$$
\phi^{2}+\mu \sigma^{2}=\mu \sigma_{\max } \sigma\left(1+\frac{\sigma^{2}}{\sigma_{c}^{2}}\right)^{1 / 2},
$$

where $\sigma_{\max }$ was given in (17) and $\sigma_{c} \equiv 2 m^{2} / \sqrt{\lambda}$. At small $\sigma$ this boundary looks like an ellipse, but at large $\sigma$ it becomes a straight line. For $\sigma_{\max }<\sigma_{c}$ these two parts of the Planck boundary are disconnected: both lines cross the $\sigma$ axis at different points. For $\sigma_{\max }>\sigma_{c}$ these two lines form a continuous curve. The greatest values of the Hubble parameter appear along the part of the Planck boundary with $\sigma \rightarrow \infty$. Therefore in this model there always exist runaway solutions describing the probability distribution $P_{p}(\phi, \sigma, t)$ rapidly moving towards indefinitely large values of $\sigma$ and $\phi$. In other words, the existence of a stationary regime which we have found in the previous model appears to be unstable with respect to a small modification of the effective potential of the inflaton field. It makes this model less attractive.

\section{One loop corrections}

Now let us return back to the simple Brans-Dicke theory (1) with the inflaton field minimally coupled to gravity, but let us take into account quantum corrections to the effective potential $V(\sigma)$. In the one-loop approximation one can represent the effective potential in the following way:

$$
V(\sigma)=\frac{\lambda}{4}\left(\sigma^{2}-\sigma_{0}^{2}\right)^{2}+\beta \sigma^{4} \ln \frac{\sigma}{\sigma_{0}},
$$

where $\sigma_{0}^{2}=m^{2} / \lambda$ is the value of the inflaton field at the minimum of the potential, and $\beta$ depends on the values of coupling constants in the theory. For example, if the scalar field $\sigma$ interacts with the fermion field with the coupling constant $h$, one obtains $\beta=\left(9 \lambda^{2}-4 h^{2}\right) / 32 \pi^{2}$ [26]. Note, that $\beta$ will be negative for $h^{2} \gg \lambda^{2}$. In that case, a new maximum will appear at exponentially 
large $\sigma$,

$$
\sigma_{\max } \simeq \sigma_{0} \exp \frac{\lambda}{4|\beta|} \gg \sigma_{0},
$$

followed by a very sharp fall-off to negative values of the potential, rendering the vacuum unstable 26. In our case this sharp fall-off may act effectively as a natural boundary for the diffusion of the inflaton field $\sigma$. In such a situation the probability distribution $P_{p}(\phi, \sigma, t)$ becomes stationary, with the maximum concentrated near the Planck boundary at $\sigma=\sigma_{\max }$.

In the theory (28), the equations of motion for $\sigma \gg \sigma_{0}$ and $\xi=0$, become

$$
\begin{aligned}
& \dot{\phi}=\frac{H \phi}{\omega}, \\
& \dot{\sigma}=-\frac{V^{\prime}(\sigma)}{3 H} \simeq-\frac{H \phi^{2}}{\omega \sigma}, \\
& H^{2}=\frac{4 \omega V(\sigma)}{3 \phi^{2}} .
\end{aligned}
$$

For $\sigma_{0} \ll \sigma \ll \sigma_{\max }$ the classical motion is circular, just as in the theory $\lambda \sigma^{4}$, and we can define polar coordinates $(r, z)$ as usual, with $z=\phi / \sigma$. Therefore one can obtain the following estimate of the Planck mass after the end of inflation:

$$
M_{\mathrm{P}} \sim \sqrt{\frac{2 \pi}{\omega}} \sigma_{\max } \sim \sqrt{\frac{2 \pi}{\omega}} \sigma_{0} \exp \frac{\lambda}{4|\beta|} .
$$

Note that in this case the effective Planck mass for $|\beta| \ll \lambda$ becomes exponentially large. In the regime when it is much greater than $\sigma_{0}$ the amplitude of density perturbations is given by the standard expression $\frac{\delta \rho}{\rho} \sim 10^{2} \sqrt{\lambda}$. Thus, one does not get anything new from the point of view of density perturbations, but one can obtain a natural explanation of the very large value of the Planck mass as compared with other mass scales in the theory.

\section{Non-perturbative effects}

All results obtained in the present paper are related to the probability distribution $P_{p}(\phi, \sigma, t)$, which shows the fraction of the volume of the Universe with the fields $\phi$ and $\sigma$ at a given moment of time $t$ in synchronous coordinates. We should emphasize again that this is not a unique choice of measure in quantum cosmology. One could study, for example, the probability distribution $P_{p}(\phi, \sigma, \tau)$, where $\tau \sim \ln a(t)$. This distribution is considerably different from $P_{p}(\phi, \sigma, t)$. This does not allow us to make unambiguous predictions until the issue of measure in quantum cosmology is resolved, see a discussion of this issue in Refs. [14, 17, 16]. Still we believe that investigation of $P_{p}(\phi, \sigma, t)$ gives us very interesting information about the structure of inflationary universe. 
Recently it was shown, in the context of chaotic inflation based on the usual Einstein theory, that the main fraction of volume of the Universe in a state with a given density $\rho$ at any given moment of time $t$ is concentrated near the centers of deep exponentially wide spherically symmetric holes in the density distribution [21]. For the reason discussed above, interpretation of this result is not unambiguous, and we are not sure that it implies that we must live near the center of a spherically symmetric void. However, we think that this result is very nontrivial and deserves further investigation [27].

Here we would like to study whether a similar effect occurs in the context of the Brans-Dicke theory. As we will see, the effect does take place, but its amplitude is significantly different. In order to understand it, we should briefly remind the origin of this effect in the Einstein theory, with one scalar field $\sigma$ (the inflation field), and then we will make a generalization to the BransDicke case.

The best way to examine this scenario is to investigate the probability distribution $P_{p}(\sigma, t)$. The distribution $P_{p}(\sigma, t)$ obeys the following diffusion equation (see Ref. [14] and references therein):

$$
\frac{\partial P_{p}}{\partial t}=\frac{1}{2} \frac{\partial}{\partial \sigma}\left(\frac{H^{3 / 2}(\sigma)}{2 \pi} \frac{\partial}{\partial \sigma}\left(\frac{H^{3 / 2}(\sigma)}{2 \pi} P_{p}\right)+\frac{V^{\prime}(\sigma)}{3 H(\sigma)} P_{p}\right)+3 H(\sigma) P_{p} .
$$

Here we temporarily use the system of units $M_{\mathrm{P}}=1$. One may try to obtain solutions of equation (32) in the form of the series $P_{p}(\sigma, t)=\sum_{s=1}^{\infty} e^{\lambda_{s} t} \pi_{s}(\sigma)$. In the limit of large time $t$ only the term with the largest eigenvalue $\lambda_{1}$ survives, $P_{p}(\sigma, t)=e^{\lambda_{1} t} \pi_{1}(\sigma)$. The function $\pi_{1}$ in the limit $t \rightarrow \infty$ has a meaning of a normalized time-independent probability distribution to find a given field $\sigma$ in a unit physical volume, whereas the function $e^{\lambda_{1} t}$ shows the overall growth of the volume of all parts of the Universe, which does not depend on $\sigma$ in the limit $t \rightarrow \infty$. In this limit one can write Eq. (32) in the form

$$
\frac{1}{2} \frac{\partial}{\partial \sigma}\left(\frac{H^{3 / 2}(\sigma)}{2 \pi} \frac{\partial}{\partial \sigma}\left(\frac{H^{3 / 2}(\sigma)}{2 \pi} \pi_{1}(\sigma)\right)\right)+\frac{\partial}{\partial \sigma}\left(\frac{V^{\prime}(\sigma)}{3 H(\sigma)} \pi_{1}(\sigma)\right)+3 H(\sigma) \cdot \pi_{1}(\sigma)=\lambda_{1} \pi_{1}(\sigma)
$$

In the simplest theory with $V(\sigma)=\frac{\lambda}{4} \sigma^{4}$ and $H=\sqrt{\frac{2 \pi \lambda}{3}} \sigma^{2}$, Eq. (33) reads [14:

$$
\pi_{1}^{\prime \prime}+\pi_{1}^{\prime}\left(\frac{6}{\lambda \sigma^{5}}+\frac{9}{\sigma}\right)+\pi_{1}\left(\frac{6}{\lambda \sigma^{6}}+\frac{15}{\sigma^{2}}+\frac{36 \pi}{\lambda \sigma^{4}}-\frac{\lambda_{1}}{\pi \sigma^{6}}\left(\frac{6 \pi}{\lambda}\right)^{3 / 2}\right)=0 .
$$

This equation can be solved both analytically and numerically. The result is that the eigenvalue $\lambda_{1}$ is given by $d(\lambda) H_{\max }$. Here $d(\lambda)$ is the fractal dimension, which approaches 3 in the limit $\lambda \rightarrow 0$, while $H_{\max }$ is the maximum possible value of the Hubble constant during inflation, which in our case corresponds to its value at the Planck boundary $V(\sigma)=M_{\mathrm{P}}^{4}=1$. This gives $H_{\max }=2 \sqrt{\frac{2 \pi}{3}}$. Thus, in the small $\lambda$ limit one has $\lambda_{1}=3 H_{\max }=2 \sqrt{6 \pi} \approx 8.68$. 
The distribution $\pi_{1}$ depends on $\sigma$ very sharply. One can easily check that at small $\sigma$ the leading terms in Eq. (34) are the second and the last ones. (This means, in particular, that the diffusion terms in Eqs. (32), (33) can be neglected.) Therefore the solution of equation (34) for small $\sigma$ corresponding to the last stages of inflation is given by

$$
\pi_{1} \sim \sigma^{\sqrt{\frac{6 \pi}{\lambda}}} \lambda_{1}
$$

This is an extremely strong dependence. For example, $\pi_{1} \sim \sigma^{10^{8}}$ for the realistic value $\lambda \sim 10^{-13}$.

Consider all inflationary domains which contain a given field $\sigma$ at a given moment of time $t$. Let us try to find a typical value of this field in those domains at the earlier moment $t-H^{-1}$. In order to do it, one should add to $\sigma$ the value of its classical drift $\dot{\sigma} H^{-1}$. One should also add the amplitude of quantum jumps $\Delta \sigma$. The usual estimate of the magnitude of a typical jump is $\pm \frac{H}{2 \pi}$. This is a correct estimate if we are interested in a typical amplitude of jumps at any given point. However, if we are considering all domains with a given $\sigma$ and trying to find all those domains from which the field $\sigma$ could originate, the answer for may be quite different. The total volume of all domains with a given field $\sigma$ at any moment of time $t$ strongly depends on $\sigma: P_{p}(\sigma) \sim \pi_{1}(\sigma) \sim \sigma^{\sqrt{\frac{6 \pi}{\lambda}}} \lambda_{1}$, see Eq. (35). This means that the total volume of all domains which could jump towards the given field $\sigma$ from the value $\sigma+\Delta \sigma$ will be enhanced by a large additional factor $\frac{P_{p}(\sigma+\Delta \sigma)}{P_{p}(\sigma)} \sim\left(1+\frac{\Delta \sigma}{\sigma}\right)^{\sqrt{\frac{6 \pi}{\lambda}} \lambda_{1}}$. On the other hand, the probability of large jumps $\Delta \sigma$ is suppressed by the Gaussian factor $\exp \left(-\frac{2 \pi^{2} \Delta \sigma^{2}}{H^{2}}\right)$. The product of these two factors has a sharp maximum at $\Delta \sigma=\lambda_{1} \sigma \cdot \frac{H}{2 \pi}$. In other words, most of the domains of a given field $\sigma$ are formed due to the jumps which have definite sign (they are decreasing the value of the scalar field), and which are greater than the "typical" ones by the amplification factor $N=\lambda_{1} \sigma$. In the usual notation, this amplification factor is given by

$$
N=\frac{\lambda_{1} \sigma}{M_{\mathrm{P}}^{2}}
$$

where $\lambda_{1}=3 H_{\max }=2 \sqrt{6 \pi} M_{\mathrm{P}} \approx 8.68 M_{\mathrm{P}}$. For $\sigma \sim 4.5 M_{\mathrm{P}}$ (the scale at which the large scale structure of our part of the Universe has been formed), the amplification factor in our theory is about 40. However, the value of this factor is very sensitive to our assumptions concerning the Planck boundary, it can be much bigger or much smaller than 40. As explained in [21], this effect does not alter the standard theory of density perturbations in inflationary universe, but it puts these perturbations on the top (or, more precisely, to the bottom) of the distribution of the scalar field $\sigma$ which appears as a result of its large jumps.

We can now return to the Brans-Dicke theory with $\omega \gg 1$. In this case both fields $\phi$ and $\sigma$ move and fluctuate, and therefore one should write a two-dimensional diffusion equation for these fields [17]. However, at the last stages of inflation the classical motion of the scalar field $\phi$ is 
very slow, and, as we argued above, diffusion in the first approximation can be neglected. Then the problem reduces to the one we have already solved, and the amplification coefficient will be given by eq. (36). The only difference is that now the value of the coefficient $\lambda_{1}=3 H_{\max }$ will be determined not by the Planck mass at the end of inflation, but by the much smaller Planck mass at the place near the Planck boundary corresponding to the peak of the distribution $P_{p}(\phi, \sigma)$. If the distribution is stationary due to the existence of some kind of boundary at $\sigma=\sigma_{\max }$, then the peak is concentrated at the Planck boundary near $\sigma_{\max }$. In this case one can show that $\lambda_{1}=3 H\left(\sigma_{\max }\right)=2 \sigma_{\max } \sqrt{3 \pi \sqrt{\lambda}}$, for $V(\sigma)=\frac{\lambda}{4} \sigma^{4}$. Meanwhile, the typical Planck mass after inflation is given by $M_{\mathrm{P}} \sim \sqrt{\frac{2 \pi}{\omega}} \sigma_{\max }$ [17]. This leads to the following realization of Eq. (36) for the Brans-Dicke theory:

$$
N \sim(6 \omega \sqrt{\lambda})^{1 / 2} \frac{\sigma}{M_{\mathrm{P}}}
$$

which gives $N \sim 4.5 \sqrt{6 \omega \sqrt{\lambda}}$, for $\sigma \sim 4.5 M_{\mathrm{P}}$. Note that for $\lambda \sim 10^{-13}$ and $\omega \sim 10^{3}$ the factor $N$ becomes very small, and therefore all the nonperturbative effects discussed above will be negligible, contrary to the case in general relativity [21].

Note that in this investigation we have assumed the existence of the upper boundary $\sigma_{\max }$. The situation will be quite different if there were no stationary solutions for $P_{p}$. In such a case the nonperturbative effects will be extremely strong. However, then we will meet the problem of runaway solutions which would suggest that $M_{\mathrm{P}} \rightarrow \infty$ in the main part of the physical volume of the Universe. In this paper we have shown that in principle it is possible to avoid runaway solutions in the Brans-Dicke theory. But then from our results it follows that the same trick which makes the distribution stationary simultaneously kills the nonperturbative effects in the inflationary Brans-Dicke cosmology.

\section{Conclusions}

Investigation of the probability distribution $P_{p}$ gives us a lot of interesting information about the properties of the inflationary universe. Some of these properties (such as the very existence of the regime of self-reproduction and the fractal structure of the Universe) do not depend on the choice of time parametrization, and therefore their interpretation is relatively straightforward. Some other properties of $P_{p}$ do depend on the choice of time parametrization. Sometimes it is not enough to know $P_{p}$, we need to know also whether all parts of the Universe which are described by this distribution are equally well suited for existence of life. In such situations interpretation of the results becomes increasingly speculative. Nevertheless we believe that even in these cases investigation of $P_{p}$ and attempts of its interpretation can be very useful. We are learning how to 
formulate questions in the context of quantum cosmology. In some cases we are obtaining results which look obviously incorrect or contradict observational data; then we may conclude that we are using quantum cosmology in a wrong way. In some other cases quantum cosmology allows us to obtain important results which cannot be obtained by other methods. This may be considered as an indication that we are on the right track. Thus, by this trial and error method we may finally learn how to use quantum cosmology.

To give a particular example one may consider the old issue of the wave function of the Universe. The two most popular candidates are the Hartle-Hawking wave function, which in the context of inflationary cosmology reads $\exp \left(\frac{3 M_{\mathrm{P}}^{4}}{16 V(\phi)}\right)$ [28], and the tunneling wave function $\exp \left(-\frac{3 M_{\mathrm{P}}^{4}}{16 V(\phi)}\right)$ [29. Neither of these two functions was rigorously derived. For some reason which is not related to its derivation, the square of the Hartle-Hawking wave function correctly describes tunneling between two different de Sitter universes with different values of $V(\phi)$ 30. However if one makes an attempt to apply it to the probability of creation of the inflationary universe, one comes to a physically incorrect conclusion that it is much easier to create an infinitely large inflationary universe with $V(\phi) \rightarrow 0$ than a Planck-size universe with $V(\phi) \sim M_{\mathrm{P}}^{4}$. The tunneling wave function leads to a qualitatively correct description of quantum creation of the Universe "from nothing," but one should not uncritically apply it to, e.g. the formation of black holes [31. The reasons for the limited applicability of each of these functions are explained in Ref. [1], whereas in Ref. [14] it is shown that expressions of the type of $\exp \left(\frac{3 M_{\mathrm{P}}^{4}}{16 V(\phi)}\right)$ or $\exp \left(-\frac{3 M_{\mathrm{P}}^{4}}{16 V(\phi)}\right)$ appear in many problems of quantum cosmology which are not related in any obvious way to the original "derivations" of the Hartle-Hawking and tunneling wave functions.

Something similar may occur with our investigation of the probability distribution $P_{p}$. This distribution is certainly very useful, but sometimes it becomes tempting to use it in the situations where its interpretation is ambiguous and the final success is not guaranteed. However, the possibility to obtain very strong results and to look at the interplay between particle physics and cosmology from an entirely new point of view suggests us to continue this trial-and-error investigation. Here we may mention our attempt to address the cosmological constant problem in the context of the Starobinsky model [16] and the possibility to explain why in the main part of the Universe the scalar-tensor theories of gravity are reduced to the Einstein theory [32]. In this paper (see also our previous publications [13, 17, 18]) we demonstrated that in the context of inflationary Brans-Dicke theory it may be the possible to explain the anomalously large value of the Planck mass $M_{\mathrm{P}}$. We have shown also that the structure of our part of the Universe which could appear as a result of nonperturbative effects in quantum cosmology [21] may be extremely sensitive to the properties of the theory at nearly Planckian densities and to the presence or absence of stationary solutions for the distribution $P_{p}$. At the very least, what we have found 
can be considered as a description of rather nontrivial properties of hypersurfaces of a given synchronous time in the inflationary universe. However, we hope that some of our results may have deeper physical significance.

\section{Acknowledgements}

J.G.-B. is supported by a PPARC postdoctoral fellowship. He would like to thank the warm hospitality and financial support of the Theoretical Astrophysics Group at Fermilab, where part of this work was developed. The work by A.L. was supported in part by NSF grant PHY-8612280. 


\section{References}

[1] A.D. Linde, Particle Physics and Inflationary Cosmology (Harwood, Chur, Switzerland, 1990).

[2] J.R. Gott, III, Nature 295, 304 (1982); J.R. Gott, III, and T.S. Statler, Phys. Lett. B136, 157 (1984); M. Sasaki, T. Tanaka, K. Yamamoto, and J. Yokoyama, Phys. Lett. B317, 510 (1993); M. Bucher, A.S. Goldhaber, and N. Turok, "An Open Universe From Inflation," Princeton University preprint PUPT-1507, hep-ph/9411206 (1994); K. Yamamoto, T. Tanaka, and M. Sasaki, Phys. Rev. D15 (1995), pp. 2968 and 2979.

[3] A.D. Linde, Nucl. Phys. B372, 421 (1992).

[4] A.D. Linde, "Inflation with Variable $\Omega$," Stanford University preprint SU-ITP-95-5 (1995), hep-th/9503097, to apper in Phys. Lett. B.

[5] P. Jordan, Nature (London) 164 (1949) 637; Z. Phys. 157, 112 (1959); C.H. Brans and R.H. Dicke, Phys. Rev. 124, 925 (1961); R.H. Dicke, Phys. Rev. 125, 2163 (1962); C.H. Brans, Phys. Rev. 125, 2194 (1962).

[6] B. Campbell, K. Olive and A. Linde, Nucl.Phys. B355, 146 (1991).

[7] J. García-Bellido and M. Quirós, Nucl. Phys. B368, 463 (1992).

[8] T. Damour and A.M. Polyakov, Nucl. Phys. B423, 532 (1994); T. Damour and A.M. Polyakov, "String Theory and Gravity", Report gr-qc/9411069 (1994).

[9] D. La and P.J. Steinhardt, Phys. Rev. Lett. 62, 376 (1989).

[10] P.J. Steinhardt and F.S. Accetta, Phys. Rev. Lett. 64, 2740 (1990); J.D. Barrow and K. Maeda, Nucl. Phys. B341, 294 (1990); J. García-Bellido and M. Quirós, Phys. Lett. B243, 45 (1990).

[11] A.R. Liddle and D.H. Lyth, Phys. Lett. B291, 391 (1992).

[12] R. Crittenden and P.J. Steinhardt, Phys. Lett. B293, 32 (1992).

[13] A.D. Linde, Phys. Lett. B238, 160 (1990).

[14] A.D. Linde and A. Mezhlumian, Phys. Lett. B307, 25 (1993); A.D. Linde, D.A. Linde, and A. Mezhlumian, Phys. Rev. D49, 1783 (1994).

[15] A. Vilenkin, Phys. Rev. Lett. 74, 846 (1995). 
[16] J. García-Bellido and A.D. Linde, Phys. Rev. D51, 429 (1995).

[17] J. García-Bellido, A.D. Linde, and D.A. Linde, Phys. Rev. D50, 730 (1994).

[18] J. García-Bellido, Nucl. Phys. B423, 221 (1994).

[19] A.D. Linde, Phys. Rev. D49, 748 (1994).

[20] A.M. Laycock and A.R. Liddle, Phys. Rev. D49, 1827 (1994).

[21] A.D. Linde, D.A. Linde and A. Mezhlumian, Phys. Lett. B345, 203 (1995).

[22] C.M. Will, Theory and Experiment in Gravitational Physics (Cambridge U.P., 1993).

[23] J.A. Casas, J. García-Bellido and M. Quirós, Phys. Lett. B278, 94 (1992); F.S. Accetta, L.M. Krauss and P. Romanelli, Phys. Lett. B248, 146 (1990).

[24] A.A. Starobinsky, J. Yokoyama, "Density Fluctuations in Brans-Dicke Inflation", preprint astro-ph/9502002, to appear in the proceedings of the Fourth Workshop on General Relativity and Gravitation, eds. K. Maeda et. al., Kyoto, Japan (1994).

[25] J.A. Casas, J. García-Bellido and M. Quirós, Nucl. Phys. B361, 713 (1991); L.J. Garay and J. García-Bellido, Nucl. Phys. B400, 416 (1993).

[26] I.V. Krive and A.D. Linde, Nucl. Phys. B117, 265 (1976).

[27] A.D. Linde, D.A. Linde, and A. Mezhlumian, in preparation.

[28] A. Vilenkin, Phys. Lett. B117, 25 (1982); J.B. Hartle and S.W. Hawking, Phys. Rev. D28, 2960 (1983).

[29] A.D. Linde, JETP 60, 211 (1984); Lett. Nuovo Cim. 39, 401 (1984); Ya.B. Zeldovich and A.A. Starobinsky, Sov. Astron. Lett. 10, 135 (1984); V.A. Rubakov, Phys. Lett. 148B, 280 (1984); A. Vilenkin, Phys. Rev. D30, 549 (1984).

[30] A. Starobinsky, in H.J. de Vega and N. Sanchez, Eds., Current Topics in Field Theory, Quantum Gravity and Strings, Lecture Notes in Physics 206, (Heidelberg: Springer)(1986); A. Goncharov and A. Linde, Sov. J. Part. Nucl. 17, 369 (1986); A. Linde, Nucl. Phys. B372, 421 (1992).

[31] S.W. Hawking and S.F. Ross, "Duality between Electric and Magnetic Black Holes", preprint DAMTP/R-95/8, hep-th/9504019 (1995).

[32] J. García-Bellido and D. Wands, "General Relativity as an Attractor of Scalar-Tensor Stochastic Inflation", preprint SUSSEX-AST-95/3-1, gr-qc/9503049 (1995). 